\title{
Why Planck (the Satellite) could have been Zel'dovich
}

\author{
Bruce Partridge ${ }^{1 \dagger}$ \\ ${ }^{1}$ Haverford College, 370 Lancaster Ave., Haverford, PA 19041, United States \\ email: bpartrid@haverford.edu
}

\begin{abstract}
In this brief paper, I cannot provide an overall review of the Planck results to date. Instead I will focus on a handful of results from both Planck and related cosmic microwave background (CMB) experiments that reflect Ya. B. Zel'dovich's legacy in cosmology. These include the Sunyaev-Zel'dovich effect in clusters of galaxies and in the cosmic web, and a map of the overall distribution of mass in the Universe derived from CMB maps.
\end{abstract}

Keywords. cosmology, cosmic microwave background, clusters of galaxies

\section{Introduction}

This brief report is not an attempt to summarize the scientific results to date of the Planck mission (see the article by Dick Bond in this volume for further details). Instead, I have tried to select a few topics, several of them astrophysical rather than cosmological, that I believe would have interested Yakov Borisovich Zel'dovich. The selection was not easy, given his wide interests, but in the end I came up with:

(1) Planck's picture of the cosmic web at $z=1100$,

(2) the Sunyaev-Zel'dovich (SZ) effect in clusters of galaxies,

(3) other loci for the SZ effect,

(4) the cosmic infrared background (CIB; in effect, the light of the cosmic web), and

(5) gravitational lensing maps of the overall mass distribution in cosmic space.

Despite the recent interest in the imprint of early inflation on the cosmic microwave background (CMB) following the BICEP2 results (Ade, Kovacs et al. 2014), I skip over that topic since Planck's findings on that subject were not available at the time of the symposium. Instead, I have added two topics that I think might have amused Ya. B.:

(6) using Planck to calibrate ground-based radio telescopes and

(7) using Planck and related measurements to measure the mass of neutrinos.

Now to explain my title. The cosmic microwave background (CMB) has an almost perfect blackbody spectrum: hence the name Planck for the mission. But Planck (the satellite) is designed to measure anisotropies in the CMB - the seeds of cosmic structure and a subject of deep interest to Zel'dovich. Given his wide contributions to so much of the science Planck was designed to probe, the mission could well have been Zel'dovich.

\section{Planck's map of the cosmic web at $z \sim 1100$}

Images of the CMB like Fig. 1 trace the distribution of matter at the epoch of last scattering at $z \sim 1100$; they are in effect snapshots of the cosmic web at early times. This topic is too rich to treat in detail here (see many other papers in this volume). I will merely say that I regret Zel'dovich did not have the opportunity to see this map, or the equally stunning earlier one made by WMAP.

$\dagger$ In part, on behalf of the Planck Collaboration 


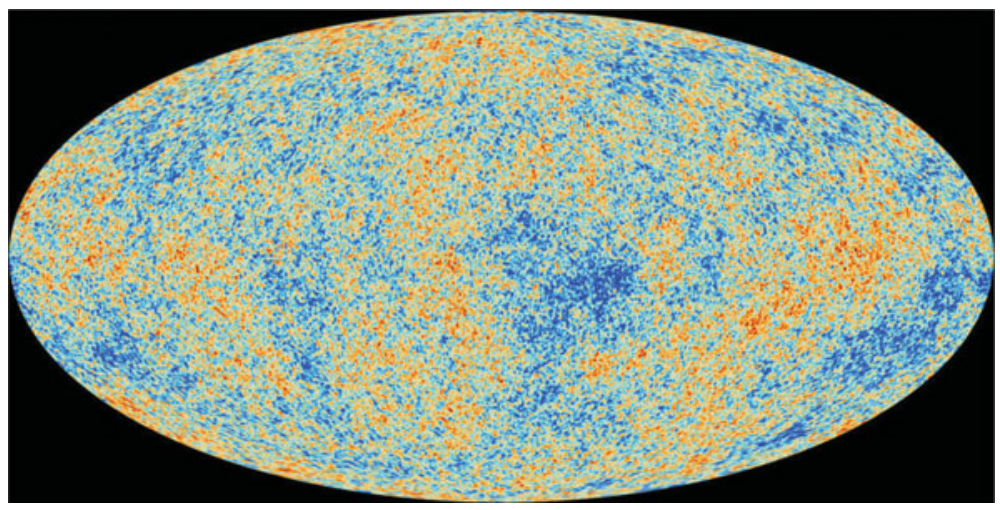

Figure 1. Planck's map (in Galactic co-ordinates); see Planck Collaboration a, 2014. The $\sim 100 \mu \mathrm{K}$ temperature variations shown in false color trace faint density perturbations that are the seeds of the cosmic web and all large scale structure.

\section{Planck's survey of clusters of galaxies using the Sunyaev-Zel'dovich Effect}

Within a few years of the discovery of the CMB, Sunyaev and Zel'dovich (see their 1980 review) had shown that inverse Compton scattering of CMB photons off hot electrons in cosmic plasmas (such as the intergalactic medium in clusters of galaxies) would affect the CMB spectrum. The Sunyaev-Zel'dovich Effect (SZE) is proportional to the pressure of hot electrons, but independent of redshift; hence it has long been recognized as a way to search for clusters at all redshifts. Both Planck and ground-based experiments like the South Pole Telescope (SPT; Bleem et al. 2014) and the Atacama Cosmology Telescope (ACT; Hasselfield et al. 2013) have made surveys of clusters using the SZE, more than doubling the number of known clusters. Planck, of course, covers the entire sky, but its broad beams limit sensitivity, so it is best at detecting massive, hot clusters at all redshifts: the 2013 Planck cluster catalog (Planck Collaboration e, 2014) contains all $\sim 1200$ of the most massive clusters in the Universe. In Fig. 2, I show an example of one of the ground-based surveys, which can reach lower masses than Planck.

The number density of clusters as a function of redshift is strongly dependent on cosmological parameters (for instance, the Dark Energy equation of state). A well-defined, mass-limited catalog of clusters thus provides a clear test of such parameters. Fig. 3 shows an example, drawn from work by the SPT Team. The crucial, and still not fully resolved, problem with this method is the scaling relation between the integrated SZE signal (Y) and the total cluster mass (see Fig. 4).

\section{Other sites for the SZE}

Clusters of galaxies are not alone in producing an SZE signal: any region of hot plasma can do so. That is true, for instance, of the hot gas entrained in the cosmic web, which Planck has mapped (Planck Collaboration d, 2014). Another site for the SZE are the gaseous halos of AGN; Gralla et al. (2014) have found this signal by stacking ACT maps at the positions of AGN. That work allows an extension of the important scaling relation between cluster mass and the SZE signal to lower masses (Fig. 4, right).

\section{The Cosmic Infrared Background (CIB)}

Galaxies trace the cosmic web, and the CIB traces galaxies, especially star-forming ones. The CIB, in a sense is the light of the cosmic web. Planck has provided detailed 


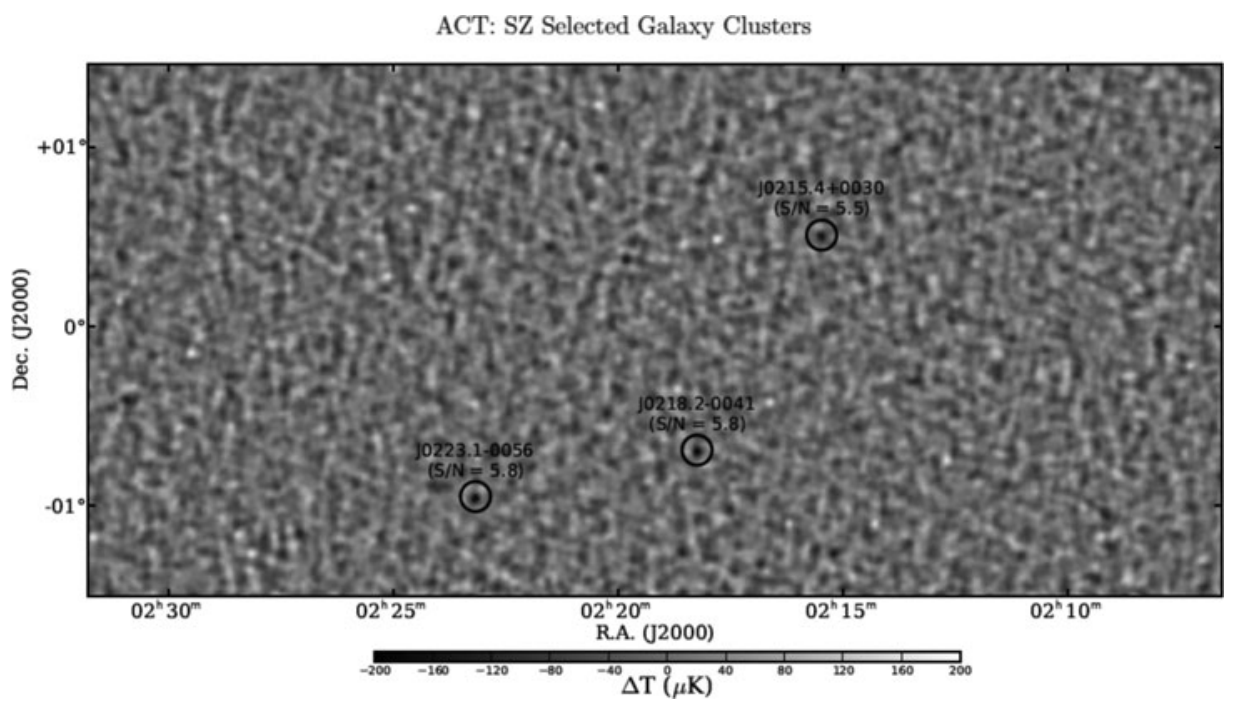

Figure 2. SZE-detected clusters in a small portion of one of the survey fields of ACT. AdvACT, its successor, will detect $\sim 27,000$ clusters in half the sky (all clusters with $M>2 \times 1014 \mathrm{Mo}$ ).

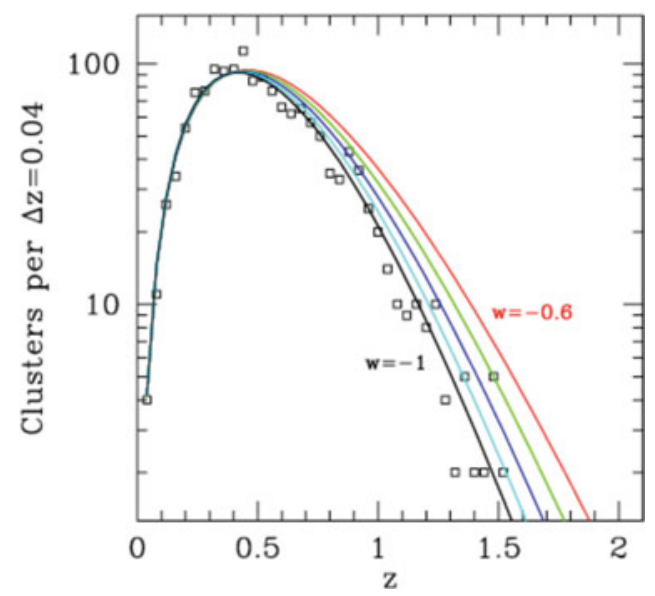

Figure 3. Counts of clusters as a function of redshift, showing the dependence on the Dark Energy equation of state parameter, $w$ (from the SPT Collaboration).

images of the CIB, as well as measurements of its intensity and power spectra (Table 1). Since the CIB spectrum is fairly sharply peaked, observations at different frequencies probe different redshifts, so Planck can supply crude tomography of the CIB.

\section{Gravitational lensing of the CMB by intervening matter}

As CMB photons move through the cosmic web, they are are gravitationally lensed by large scale structure. Measuring this lensing permits a reconstruction of the line-of-sight mass integral across sky (Fig. 5; Planck Collaboration c, 2014). Measuring this lensing also permits an estimate of the lensing power spectrum. I note that the lensing kernel has a broad maximum at $z \sim 2$, just where star formation peaks up. 


\begin{tabular}{rll}
\hline Band & \multicolumn{2}{c}{$\nu I_{\nu}\left[\mathrm{nW} \mathrm{m}^{-2} \mathrm{~s} \mathrm{r}^{-1}\right]$} \\
\hline $3000 \mathrm{GHz}$ & 13.1 & \pm 1.0 \\
$857 \mathrm{GHz}$ & 7.7 & \pm 0.2 \\
$545 \mathrm{GHz}$ & 2.3 & \pm 0.1 \\
$353 \mathrm{GHz}$ & 0.53 & \pm 0.02 \\
$217 \mathrm{GHz}$ & 0.077 & \pm 0.003 \\
\hline
\end{tabular}

Table 1. Intensity of the CIB in the IR (from Planck Collaboration f, 2014).
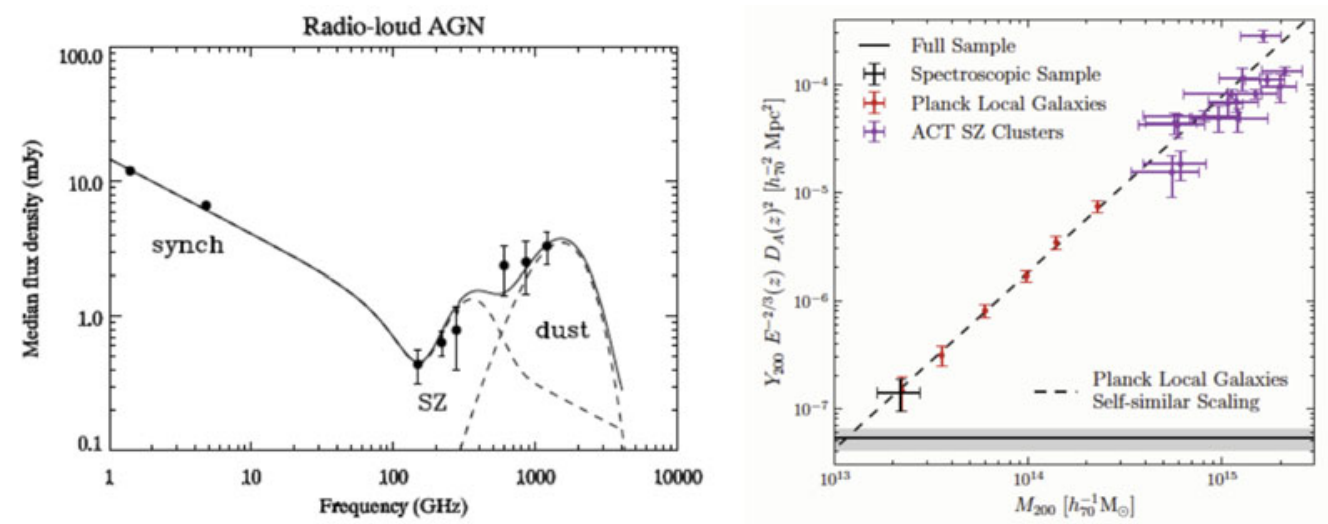

Figure 4. Evidence for the SZE in the gaseous halos of AGN; the result for low mass halos lies along the expected mass-SZE scaling relation for clusters (Gralla, et al., 2014).

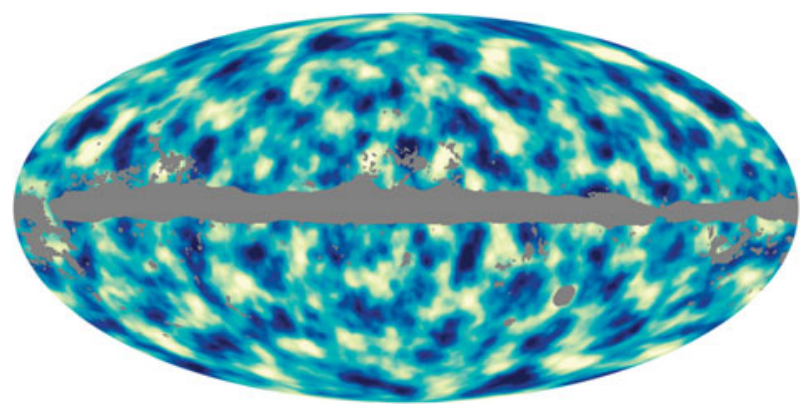

Figure 5. Lensing permits a reconstruction of the line-of-sight integral of all mass in the Universe. That integral is shown here in Galactic coordinates (Planck Collaboration c, 2014).

There is good agreement between the measured lensing power spectrum (Fig. 6) and the value predicted from $\Lambda$ CDM cosmology (showing that we know how the cosmic web evolves). If anything, the measured lensing is a bit larger than expected.

\section{Using Planck to calibrate ground-based radio telescopes}

The intensity calibration of Planck is absolute, pinned to the satellite's motion in the solar system. Furthermore, Planck is sensitive enough to observe hundreds of bright radio sources as well as planets. Comparing Planck and ground-based measurements allows us to transfer Planck's calibration to the VLA, the Australia Telescope, ALMA, etc. In the case of bright radio sources (and Mars), the variability of the sources can introduce scatter and potentially bias in the Planck comparison. We minimize both by making the Planck and ground-based observations at roughly the same time, and by including many 


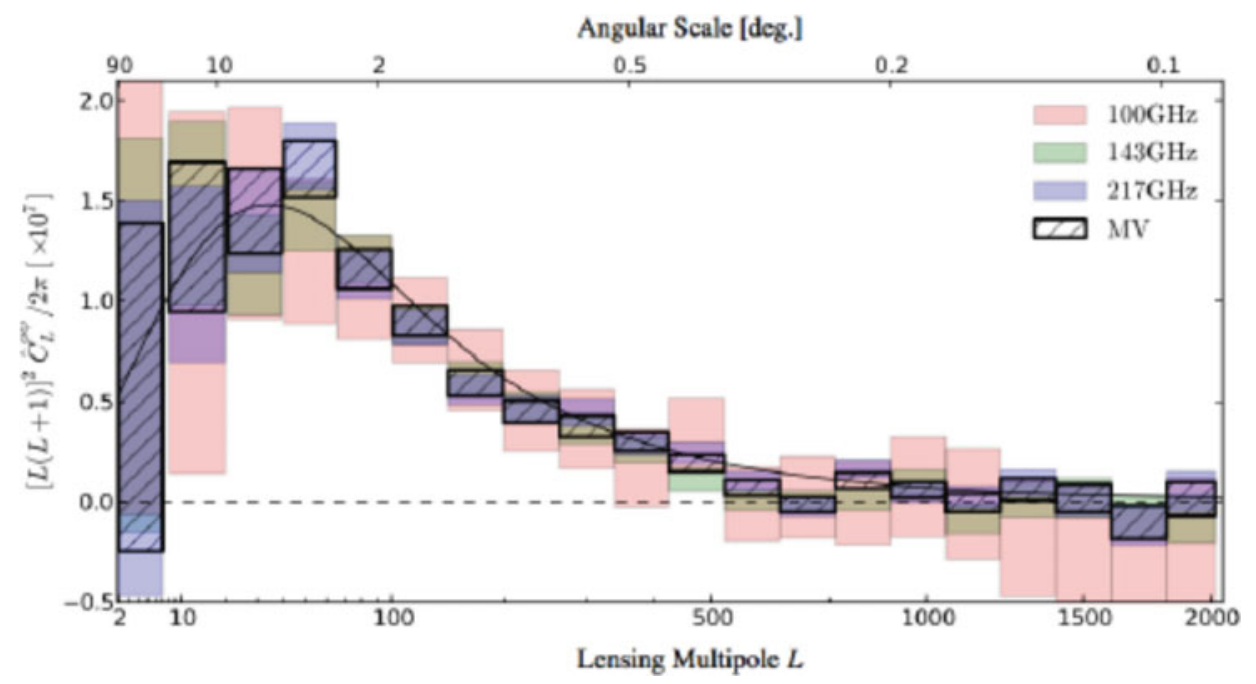

Figure 6. The lensing power spectrum measured by Planck at three frequencies (boxes) compared to the expected value derived from the cosmological parameters determined by Planck: note the excellent agreement (Planck Collaboration c, 2014).

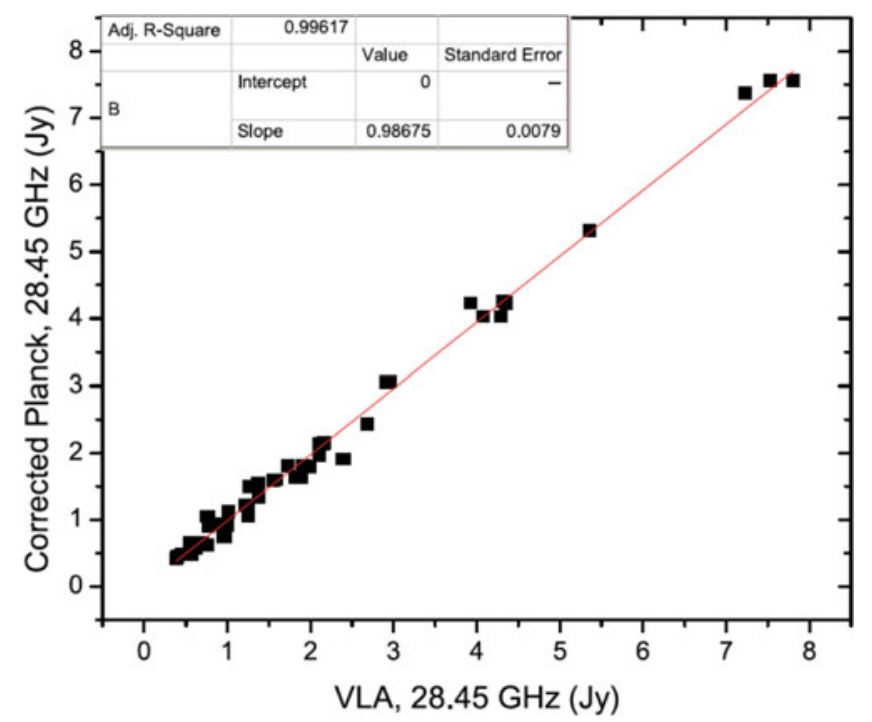

Figure 7. Flux densities of bright radio sources measured by Planck and the VLA at $28 \mathrm{GHz}$. The slope lies within $\sim 1 \%$ of unity. The scatter is dominated by variability in these radio sources. An updated version appears in Perley et al. (2015).

sources (Perley et al., 2015). An early example is shown in Fig. 7; it demonstrates that the newly adopted microwave flux density scale of Perley and Butler (2013) is consistent with the absolute Planck calibration to $\sim 1-2 \%$ precision at $28 \mathrm{GHz}$.

\section{Using Planck to measure the mass of neutrinos}

The mass, number and to some extent properties of neutrinos affect various aspects of the power spectrum of anisotropies in the CMB, including the small-angle damping tail (see, for instance, Abazajian t al., 2013). The Planck results (Planck Collaboration 


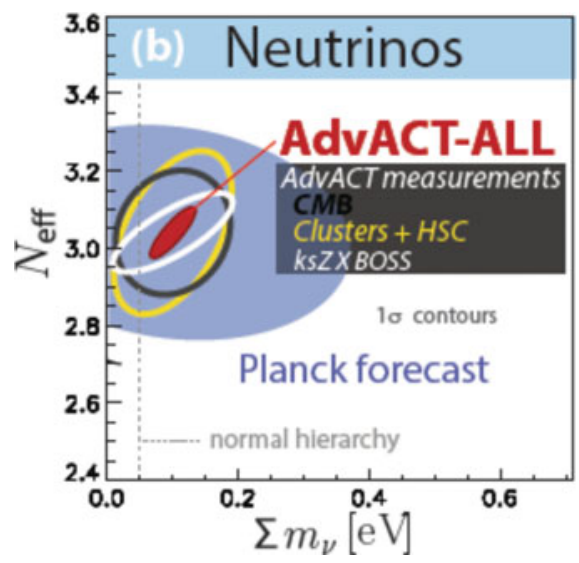

Figure 8. Constraints on neutrino masses expected from the proposed AdvACT experiment.

b, 2014), for instance, are consistent with three (not four) neutrino species, and set a limit of $<0.66 \mathrm{eV}$ on the sum of masses of these three. Adding other astrophysical data allows a lower limit to be set: $<0.23 \mathrm{eV}$. As an instance of the power of planned CMB experiments to constrain both the number and mass of neutrinos, I show below projections for AdvACTPol.

\section{Conclusions}

Much of the science I have sketched relies on our increasingly sensitive measurements of anisotropies in the CMB, including Planck's. Even though not all the topics I addressed have a direct link to the cosmic web, I hope I have helped convince you of the value and versatility of current CMB results. I know I do not have to convince you of the range and value of Ya. B. Zel'dovich's contributions to cosmology.

\section{References}

Abazajian, K. N, et al. 2013, arXiv:1309.5383.

Ade, P., Kovacs, J., et al. 2014 Phys. Rev. Letters 112, 241101.

Bleem, L. E, et al. 2014, arXiv:1409.0850.

Gralla, M., et al. 2013, MNRAS, submitted; arXiv:1310.8281.

Hasselfield, M. et al. 2013, JCAP 07, .

Perley, R.A. and Butler, B.J. 2013, ApJS 204, 19.

Perley, R. A, Partridge, B., et al. 2015 in preparation.

Planck Collaboration a 2014, Planck 2013 results I; arXiv:1303.5062.

Planck Collaboration b 2014, Planck 2013 results XVI; arXiv:1303.5076.

Planck Collaboration c 2014, Planck 2013 results XVII; arXiv:1303.5077.

Planck Collaboration d 2014, Planck 2013 results XXI; arXiv1303.5081.

Planck Collaboration e 2014, Planck 2013 results XXIX; arXiv1303.5089.

Planck Collaboration f 2014, Planck 2013 results XXX; arXiv1309.0382.

Sunyaev, R. A, and Zel'dovich, Ya. B. 1980, Ann. Rev. Astron. Astrophys. 18, 537. 